apuntesuniversitarios.upeu.edu.pe

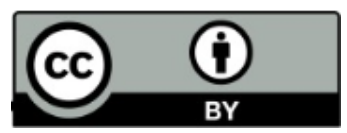

$C C-B Y$

Apuntes Universitarios, 2021: 11(4), octubre-diciembre

ISSN: 2304-0335 DOI:https://doi.org/au.v11i4.765

\title{
Las escuelas de los profetas del antiguo Israel y su impacto en la educación adventista
}

\author{
Ancient Israel's Schools of the Prophets and their impact on the Adventist Education
}

\author{
Eric E. Richter ${ }^{1 \mathrm{a}}$, Ana Maurín ${ }^{2}$ \\ Universidad Adventista del Plata, Argentina \\ iD Orcid ID https://orcid.org/0000-0002-6407-6157 ${ }^{1}$ \\ iD Orcid ID https://orcid.org/0000-0001-5887-6310²
}

Recibido: 08 de marzo 2021

Aceptado: 26 de junio de 2021

\section{Resumen}

La educación religiosa de los antiguos israelitas dependía en gran manera de las así llamadas escuelas de los profetas, cuyo establecimiento era considerado de origen divino. Estas escuelas poseían un modelo educativo caracterizado principalmente por el trato docente-alumno, el papel de la espiritualidad en la educación, la inculcación de una ética personal y el uso del trabajo manual por parte de los alumnos. En este contexto, el objetivo de este estudio es mostrar cómo el modelo pedagógico y la filosofía educativa de las escuelas de los profetas han impactado el modelo de la educación ministerial adventista. Se prestará especial atención a los escritos y rol de Ellen White, así como a otros propulsores del sistema educativo adventista. Las conclusiones sintetizarán la manera en que las escuelas de los profetas han influido en el modelo de educación ministerial adventista y los puntos en común entre ambos sistemas pedagógicos.

Palabra clave: Educación, Elena G. de White, educación cristiana, educación teológica, Iglesia Adventista del Séptimo día, Antiguo Testamento, profetas.

\begin{abstract}
The religious education of the ancient Israelites depended heavily on the so-called schools of the prophets, the establishment of which was considered to be of divine origin. These schools had an educational model characterized mainly by teacher-student treatment, the role of spirituality in education, the inculcation of personal ethics and the use of manual labor by students. Therefore, the purpose of this study is to show how the pedagogical model and educational philosophy of the schools of the prophets have impacted the Adventist model of ministerial education. Special attention will be paid to the writings and role of Ellen White, as well as other proponents of the Adventist educational system. The conclusions will summarize the way in which the schools of the
\end{abstract}

${ }^{\mathrm{a} C}$ Correspondencia al autor

E-mail: eric.richter@uap.edu.ar 
prophets have influenced the Adventist model of ministerial education and the points in common between both pedagogical systems.

Keywords: Education, Ellen G. White, Christian education, theological education, Seventh-day Adventist Church, Old Testament, prophets.

\section{Introducción}

La Iglesia Adventista del Séptimo Día considera de vital importancia la educación de niños y jóvenes, y por ello ha desarrollado un sistema educativo cristiano que es considerado el segundo más grande del mundo (Kido, 2010). Operar de manera coordinada un número elevado de escuelas, colegios y universidades exige poseer una filosofía de la educación que se desempeñe como principio guiador y base fundacional de toda actividad pedagógica. Como una denominación cristiana, la Iglesia Adventista del Séptimo Día ha buscado conformar su filosofía educativa en base de la Biblia. Esto aplica sobre todo para el entrenamiento ministerial o la educación teológica.

Entre los antecedentes bíblicos que han desempeñado un papel importante en la formación de la filosofía educativa adventista para la formación de ministros y obreros eclesiásticos, la figura de las escuelas de los profetas ha sido particularmente significativa. De hecho, varios estudios previos han explorado esta conexión. Peck (1992), por ejemplo, considera que son un modelo indispensable a seguir para establecer seminarios de educación teológica. Su propuesta busca contextualizar los principios pedagógicos de las escuelas de los profetas a la situación educativa Rwanda. Por su parte, Millanao-Orrego (1992) procuró mostrar cómo este modelo pedagógico puede ser aplicado para las instituciones ministeriales de Sudamérica. Hong (2000) afirma que las escuelas de los profetas son el paradigma clave para la educación cristiana, incluida la ministerial o teológica, en el sistema educativo adventista en Korea. En tanto Appiah et al. (2017) procuró contextualizar estos principios para la situación educativa de Ghana.

Otros autores recientes también han explorado el tema. Por ejemplo, Davidson (2015) considera que las escuelas de los profetas son el paradigma fundamental para la educación teológica y ministerial. Por su parte, Taylor (2017) ha sugerido que sus postulados educativos básicos deben ser imitados al establecer una filosofía educativa adventista. En tanto Tayo et al. (2019) han indicado que las escuelas de los profetas presentan la fusión ideal entre educación formal y espiritualidad personal, y, por este motivo, son un ejemplo a seguir para la educación superior cristiana. 
El presente artículo se propone indagar cuáles eran las características de las escuelas de los profetas, la manera en que este modelo fue introducido dentro de la filosofía de la educación, y cuáles principios de este sistema pedagógico pueden ser aplicados en la conformación de un modelo adventista de educación ministerial y teológica.

Con este fin en mente, se realizará una investigación de carácter documental. La metodología a seguir incluirá tres pasos. En primer lugar, se indagará lo que las fuentes históricas declaran acerca de las características de las escuelas de los profetas del Antiguo Israel. Luego se mostrará la manera en que Ellen White, la principal promotora de la educación adventista en el siglo XIX, percibió estas escuelas y los elementos que tomó como inspiración en la conformación del modelo educativo adventista. En tercer lugar, se recapitularán los principios básicos propios del sistema educativo de las escuelas de las escuelas de los profetas que pueden ser aplicados en la formación de una filosofía adventista de la educación ministerial y teológica. Se concluirá con algunas reflexiones finales.

\section{Las escuelas de los profetas en el antiguo Israel}

Además de las obras que componen la Biblia Hebrea o el Antiguo Testamento, existen pocas fuentes históricas que proporcionen información relevante sobre el Antiguo Israel. El enfoque minimalista de la investigación arqueológica y la metodología histórica-crítica del estudio exegético han arrojado dudas acerca de la confiabilidad del relato bíblico, pero diversos autores han presentado argumentos convincentes en favor de la historicidad del Antiguo Testamento, entre ellos Kaiser Jr. (2001), Kitchen (2003), Provan et al. (2015) y Berman (2017).

Por lo tanto, el testimonio bíblico bien puede aportar una imagen verosímil y fidedigna de la vida cotidiana del Antiguo Israel. Esta afirmación es de especial relevancia, pues solo la Biblia Hebrea presenta información acerca de las escuelas de los profetas. Aunque es posible que algunas inscripciones hebreas antiguas hayan pertenecido a escuelas israelitas, no hay manera de confirmar que fueron utilizadas en escuelas de los profetas. Además, sea cual sea el caso, no proporcionan información relevante acerca de ellas (Lemaire, 1981). Debe notarse, sin embargo, que la frase "escuela de los profetas" no aparece textualmente en el Antiguo Testamento, aunque si es posible encontrar expresiones similares, como "hijos de los profetas" (cf. 1 Re 20:35; 2 Re 2:3, 5, 7, 15; $4: 1,38 ; 5: 22 ; 6: 1 ; 9: 1)$ o “compañía de profetas” (1 Sam 10:5; 19:20). 
El origen exacto de estas escuelas está envuelto en el misterio, aunque la tradición judía consideraba al profeta Samuel como el fundador de estas instituciones (Sicker, 2011). Es posible que en sus inicios el profeta se desempeñara como maestro utilizando su residencia personal en Ramá como instalación educativa. Wood afirma que:

Hay un pasaje que nos da la clave para barruntar que también la escuela de Samuel disponía de un edificio como centro de instrucción: En 1 S.19:18,19 se nos dice que David había huido a donde estaba Samuel con sus profetas en "Nayot en Ramá". La palabra nayoth significa "habitaciones" o "edificios". Esto puede dar el sentido de que David se fue hacia Samuel, quien estaba en un edificio de dicha escuela, situado en Ramá, donde Samuel tenía su casa. (1990, p. 172)

Las menciones de "hijos de los profetas" en diferentes ubicaciones permite inferir que se levantaron nuevas escuelas de los profetas en localidades como Betel y Jericó (2 Re 2:1-5), además de Gilgal (2 Re 4:38-41), Quiriat Jearim (Jer 26:20), Samaria (2 Re 6:1) y Jerusalén (2 Re 22:14). Cada sede estaba ubicada en sitios estratégicos, ya sea como paso de rutas comerciales o lugares de frontera (Quinteros, 2013). Jericó, por ejemplo, era atravesado por varias rutas comerciales antiguas. Samaria y Jerusalén eran las capitales del Reino de Israel y de Judá respectivamente. Betel y Gilgal era centros de adoración idolátrica. Jericó era un lugar de paso en varias rutas comerciales. Rama era parte de la frontera entre el reino de norte y el reino del sur. Además, Jerusalén, Gilgal y Quiriat-Jearim tenían gran significancia religiosa para la historia hebrea. Estas ubicaciones permitían que los estudiantes tuvieran contacto con e influyeran a gran cantidad de personas.

Las escuelas contaban con líderes educativos, como fue el caso de Samuel, Elías, Eliseo, Hulda o Isaías, que solían también supervisar varias sedes escolares. No era raro que los alumnos vivieran junto a sus maestros, como fue el caso de Samuel (1 Sam 7:17) y Elías (2 Re 6:1). Aunque quienes eran casados y tenían su propia familia solían vivir por su propia cuenta $(2 \operatorname{Re} 4: 1)$. Como los profetas/maestros solían vivir junto a los estudiantes $\mathrm{y}$, en ocasiones, viajar con ellos, la atención que recibían era personalizada. La relación cercana que existía entre los maestros y sus discípulos permitía que estos acudieran a ellos no solo por instrucción religiosa, sino también en busca de orientación o ayuda en caso de problemas personales (ver por ejemplo el caso de 2 Re 4:1-7). Es posible que esta relación paternal esté detrás del nombre más común que recibían estos estudiantes: "hijos de los profetas" (Williams, 1966; Estep, 2003). 
En ocasiones, el profeta elegía a uno de sus discípulos para que sea su sucesor. Esta preparación requería una atención personalizada mayor en la cual el alumno servía a su maestro como a un señor. En estos casos se puede mencionar a Elías con Eliseo, y a este último con Giezi. Es posible que el caso de Jeremías con Baruc haya seguido esta línea (Jer 36:4-10), aunque esto no es seguro.

Los estudiantes también desempeñaban un papel importante en el mantenimiento de sus escuelas. Solían participar en la construcción o ampliación de los edificios (2 Re 6:1-7) y procuraban ser de sostén propio (2 Re 4:39). Esto implicaba que la formación que se daba en estos lugares no solo era teórica, sino también práctica. La instrucción recibida incluía también el aprendizaje y desarrollo de habilidades y oficios prácticos. También es posible que la educación incluyera el estudio de la música, pues en muchas ocasiones se menciona a los profetas ejecutando instrumentos musicales (Standish \& Standish, 1996).

Sin embargo, el énfasis de la educación era claramente religioso. Después de todo, las escuelas de los profetas eran centros de educación espiritual y ministerial que permitieran formar líderes no sacerdotales que educaran al pueblo y lo instaran a mantener su fidelidad al Dios de Israel. En palabras de Dyrness (1977), "they were proclaimers of the word of the Lord" (p. 215).

De acuerdo a los relatos bíblicos, la educación impartida en las escuelas de los profetas sirvió para capacitar a numerosos jóvenes que desempeñaron un rol muy importante en la religión y sociedad israelita. Los profetas aconsejaron a reyes (1 Re 22), ungieron nuevos monarcas (2 Re 9:6-10) y amonestaron al pueblo (2 Re 17:13). Sin embargo, con el paso del tiempo, los profetas terminaron devaluando su ministerio. La evidencia bíblica indica que los profetas comenzaron a profetizar por dinero o para agradar al rey (Jer 5:31). Esta situación generó un descontento de profetas verdaderos, quienes incluso se negaron a ser relacionados con los hijos de los profetas: "Yo no soy profeta, ni hijo de profeta" (Am 7:14). Las características educativas básicas de las escuelas de los profetas pueden resumirse en varios puntos. Davidson (2015) señala los siguientes:

- Los alumnos debían ser consagrados, inteligentes y esforzados.

- Los maestros y líderes de las escuelas debían ser profetas versados en las Escrituras, devotos a Dios y respetados por la comunidad.

- El foco de la educación estaba en la Escritura, lo cual incluía conocimiento de las leyes divinas, música de adoración y poesía. También se realizaba una instrucción balanceada en doctrina, ética y estética con el objetivo de desarrollar el carácter y 
preparar a los estudiantes para tareas ministeriales y misioneras. También se llevaba a cabo formación práctica en habilidades físicas y manuales.

- La metodología educativa se basaba en la Biblia hebrea. La relación maestro-alumno era cercana y personalizada. Se promovía la excelencia, la cooperación, el respeto y la laboriosidad.

- Las escuelas eran ubicadas en lugares estratégicos y su alumno comprendía entre 50 a 100 alumnos, sino más. Los estudiantes vivían juntos y colaboraban en el mantenimiento y sostén de la institución mediante trabajo físico. (p. 19-22)

Todos estos puntos se han visto reflejados de una manera u otra en el desarrollo del sistema educativo adventista, tal como se mostrará a continuación.

\section{Las escuelas de los profetas en el desarrollo de la filosofía educativa adventista}

Antes de mostrar el impacto que el modelo pedagógico de las escuelas de los profetas ha tenido en la filosofía adventista de la educación teológica, se mostrará brevemente el origen del sistema educativo adventista.

La Iglesia Adventista del Séptimo Día se organizó formalmente como una denominación en el año 1863. Sin embargo, recién en 1867 se estableció la primera escuela primaria, aunque producto de una iniciativa privada. En 1872 se fundó la primera escuela denominacional, a instancias de una visión de Ellen White (Brown, 1972). Con el paso del tiempo se establecieron nuevas escuelas primarias, secundarias y terciarias en Norteamérica. A fines del siglo XIX y principios del XX la expansión alcanzó a otros continentes, con colegios abriendo en Sudamérica, Interamérica, África, Europa y Asia (Greenleaf, 2005). A partir de la década de 1920 se inició lentamente el proceso de acreditación de las universidades adventistas.

El objetivo inicial de la creación de instituciones educativos era la preparación de jóvenes para convertirse en líderes y misioneros (Knight, 2015). Sin embargo, al momento de establecer las primeras escuelas y colegios se imitó la filosofía educativa clásica predominante de ese tiempo. Elena G. de White intentó contrarrestar esto mediante la publicación en 1872 de un tratado titulado "Proper Education", donde estableció los lineamientos básicos de lo que consideraba que debía consistir la filosofía educativa adventista. Aunque en este documento no se mencionó las escuelas 
de los profetas, publicaciones subsiguientes comenzaron a mostrar que éstas eran el modelo a seguir en el establecimiento de instituciones educativas adventistas.

Dos artículos, publicados en 1882 y 1885, respectivamente comenzaron a mostrar la importancia que las escuelas de los profetas tenían para el establecimiento del sistema educativo adventista. El primero, titulado "The Schools of the Prophets", definió estas instituciones como "missionary seminaries, designed to maintain a higher standard of morals and religion" (1882, p. 1). Como tales, debían convertirse en prototipos a seguir de la clase de colegios que Dios deseaba que sean establecidos. Esta idea fue enfatizada también en su artículo de 1885, "The Schools of the Ancient Hebrews", donde Ellen White afirmó la importancia de las escuelas de los profetas como un modelo de origen divino para el establecimiento del sistema educativo adventista.

En su libro Patriarchs and Prophets, publicado originalmente en 1890, incluyó un capítulo en el cual se describían las escuelas de los profetas en detalle (p. 592-602). El propósito de estas instituciones, aseguró White, era proteger "the moral and spiritual welfare of the youth" (p. 593). Este capítulo fue revisado y republicado posteriormente en 1894, como parte del libro Christian Education (p. 61-70). Allí señaló algunas características de este modelo educativo, como la centralidad de la Escritura en el currículo escolar y la importancia dada al desarrollo de la espiritualidad. Señaló que la educación debe incluir el hábito de la oración, el aprendizaje de la música sacra y la formación en habilidades manuales y físicas. Luego escribió:

Are there not some lessons which the educators of our day might learn with profit from the ancient schools of the Hebrews? He who created man has provided for his development in body and mind and soul. Hence, real success in education depends upon the fidelity with which men carry out the Creator's plan. (p. 63)

Esto muestra que el modelo propuesto por Elena G. de White a partir de las escuelas de los profetas incluía el desarrollo del cuerpo, espíritu y mente, es decir, era primordialmente holístico. Inmediatamente luego de este párrafo sigue la que se considera la definición más importante de Elena G. de White acerca de la educación: "The true object of education is to restore the image of God in the soul" (p. 63).

Es claro que Elena G. de White veía en las escuelas de los profetas el prototipo o molde a seguir en el establecimiento de instituciones educativas. Este modelo era de origen divino y debía ser imperativo para la fundación del sistema educativo adventista por encima de cualquier otra filosofía secular: 
Shall we permit men who are called great, and yet who have been taught by the enemy of all truth, to have the education of our youth? Or shall we take the word of God as our guide, and have our schools conducted more after the order of the ancient schools of the prophets? (1894, p. 82)

En 1897, varios tratados que Ellen White había escrito sobre la temática de la educación fueron publicados juntos bajo el título Special Testimonies on Education. Allí colocó el énfasis en varios principios practicados en las escuelas de los profetas, como la centralidad del estudio de la Escritura en la educación, la importancia de contar con maestros consagrados y la práctica de actividad física.

Mas tarde, en 1901, llamó a seguir cuidosamente "the same plans that were followed in the schools of the prophets" al momento de establecer nuevas instituciones educativas (p. 139). Afirmó que "Our schools must be more like the schools of the prophets... They should be family schools, where every student will receive special help from his teachers as the members of the family should receive help in the home" (p. 152). También enfatizó que los estudiantes "shall be instructed as were those in the schools of the prophets" (441). De esta manera, confirmó la necesidad de contar con relaciones docente-alumno que sean cercanas y personales.

De particular interés resulta una carta escrita ese mismo año a Percy T. Magan y E. A. Sutherland, ambos directivos del Battle Creek College, la principal institución educativa para la formación de ministros, misioneros y obreros que poseía la Iglesia Adventista por aquel entonces. Ellen White los llamó a innovar y procurar buscar siempre los mejores métodos pedagógicos, aduciendo que esta era una característica también de las escuelas de los profetas: "You should endeavor to train the very best class of workers, who as teachers and ministers of the gospel will be able to educate others... All who now are connected with the work of education must not follow the same, same old methods. Our schools should be more after the order of the schools of the prophets" (1985, p. 200).

En 1903 también incluyó un capítulo sobre las escuelas de los profetas en su obra Education (p. 45-50). Allí aseguró que Dios había sido el autor de las escuelas de los profetas, añadiendo que los profetas eran "teachers divinely appointed" (p. 46). Uno de los objetivos de estas escuelas era proporcionar un refugio a la juventud de la corrupción mental y espiritual. Las escuelas adventistas también debían proporcionar la misma clase de amparo. Ella también afirmó que las escuelas de los profetas enseñaban la lectura de la Biblia, el hábito de la oración y la importancia del trabajo manual. Todas estas actividades debían ser enseñadas también en los colegios adventistas. 
En 1913, con la publicación de su libro Counsels to Parents, Teachers, and Students repitió la importancia de modelar los colegios adventistas a partir de las escuelas de los profetas: "In all our churches there should be schools... These schools, established in different localities, and conducted by God-fearing men or women, as the case demands, should be built on the same principles as were the schools of the prophets" (p. 168). Entre los principios básicos que caracterizaban las escuelas de los profetas y debían influir en la filosofía adventista se incluía la centralidad de la Escritura en el currículo escolar, la enseñanza de oficios prácticas y habilidades manuales, la contratación de maestros consagrados y espirituales, el estudio de la Biblia y la formación de hábitos espirituales, y el aprendizaje de la historia sagrada.

Cuando se publicó la segunda edición de su libro Gospel Workers en 1915, Ellen White escribió el siguiente consejo: "Let the older workers be educators... Let the young men feel it a privilege to study under older workers... Thus Elijah educated the youth of Israel in the schools of the prophets; and young men today are to have a similar training” (p. 101-102). Con este consejo añadió la importancia de que los obreros de experiencia actúen como mentores de los más jóvenes, imitando así la práctica de las escuelas de los profetas.

Es interesante que una búsqueda en la base de datos digital Adventist Digital Library muestra que Ellen White fue la única autora adventista que escribió acerca de la escuela de los profetas entre 1861 y 1915. Su foco en estas instituciones se debía a que las consideraba ejemplos a seguir en el establecimiento de nuevos centros educativos adventistas. Se pueden resumir varios puntos principales que adoptó a partir de las escuelas de los profetas:

En primer lugar, consideró que la filosofía adventista debe ser holística en su aproximación antropológica. El ser humano está compuesto de tres partes inseparables: el cuerpo, la mente y el espíritu. Es decir, las facetas físicas, intelectuales y espirituales de la persona. La educación debe promover el desarrollo equilibrado de estos tres aspectos del ser.

En segundo lugar, Ellen White afirmó que el foco de la educación debe estar puesto en aprender acerca de Dios. La Escritura debe poseer el punto central de la pedagogía. Esto incluye también el aprendizaje de la historia sagrada, la naturaleza creada y la música sacra. En tercer lugar, se debe promover el estudio de oficios prácticos y habilidades manuales. Esto permite a los jóvenes contar con conocimientos prácticos que le permitan ganarse la vida y, al mismo tiempo, desarrollarse físicamente. 
En cuarto y último lugar, los maestros y educadores deben ser personas consagradas, entregadas a Dios y de excelencia profesional. Deben mantener una relación cuasi familiar con sus alumnos, orientándolos y guiándolos en su desarrollo personal y crecimiento espiritual.

\section{Principios pedagógicos de las escuelas de los profetas aplicados a la educación}

Aunque los consejos de Ellen White establecieron las bases del sistema educativo adventista, la sistematización de la filosofía educativa dentro de un marco académico se llevó a cabo bien entrado en el siglo XX. Quizás uno de los pioneros en la formulación académica en este campo fue George R. Knight, cuyas numerosas obras han abordado en detalle los matices filosóficos de la educación adventista (Matthews, 2014). ${ }^{1}$

Al momento de buscar las bases filosóficas de la educación adventista, Knight depende en gran medida de los consejos de Ellen White. Afirma incluso que sus escritos son "fundamentales" a la hora de definir una filosofía adventista de la educación (2012, p. 25). Como se ha mostrado anteriormente, muchos de los postulados básicos del modelo pedagógico adventista propuesto por Ellen White se basan en las escuelas de los profetas. Y es a través de los postulados de White que Knight acepta principios provenientes originalmente de estas escuelas. Por ejemplo, Knight (2012) defiende una antropología holística, que implica el desarrollo equilibrado del cuerpo, la mente y el espíritu; el énfasis espiritual y salvífico de la educación; la necesidad de maestros consagrados y calificados; etc. Todos estos principios, derivados de la filosofía educativa de Ellen White, tienen su origen en las escuelas de los profetas.

Pero los principios pedagógicos de las escuelas de los profetas, no solo han influido en la filosofía educativa en general. La Iglesia Adventista ha buscado que la capacitación ministerial y teológica esté en armonía con los principios educativos bíblicos. Becerra (2008), por ejemplo, menciona algunos postulados básicos en la educación teológica adventista que, en última instancia, tienen su origen en el modelo de las escuelas de los profetas. Por ejemplo, la importancia de una comprensión holística del ser humano lleva a descartar una dicotomía entre la razón y el físico, o

\footnotetext{
${ }^{1}$ Las principales obras de Knight que han procurado establecer las bases filosóficas del modelo de educación adventista son Philosophy and Education: An Introduction in Christian Perspective (1982), Issues and Alternatives in Educational Philosophy (1982) y Myths in Adventism: An Interpretive Study of Ellen White, Education, and Related Issues (1985). Nótese que pasó más de un siglo desde el establecimiento de la primera institución educativa adventista denominacional y comienzo de la formulación de una filosofía académica de la educación adventista. Esto manifiesta la necesidad de continuar profundizando este tema.
} 
entre lo intelectual y lo pastoral. La capacitación teológica debe enfocarse en formar y perfeccionar el todo de la persona para que de esa manera pueda brindar un mejor servicio a Dios y a sus semejantes. Plaatjes (2014), por su parte, recalca la importancia del énfasis salvífico de la educación cristiana. Afirma que la función de la educación adventista es preparar individuos que vivan de acuerdo a la voluntad de Dios y trabajen por la salvación de otros.

Existen otros principios pedagógicos de las escuelas de los profetas que son aplicados en la formación ministerial adventista, aunque contextualizados a las exigencias académicas actuales. De los principios pedagógicos que Davidson (2015) enumera, se pueden mencionar los siguientes. En primer lugar, las universidades adventistas procuran que los maestros y profesoras sean personas de aptitud espiritual, experiencia pastoral y excelencia académica (Orwenyo, 2014). Además, el foco de la capacitación o educación ministerial está puesto en la Escritura, lo cual incluye la teología bíblica, música sacra, ética cristiana, formación espiritual y preparación pastoral/misionera (IBMTE, 2017). De la misma manera, se promueve la excelencia, la cooperación, el respeto y la laboriosidad (IBMTE, 2017).

Debe mencionarse también, que algunos de los principios básicos de las escuelas de los profetas no han sido alcanzados o aplicados completamente en el sistema educativo adventista de la formación ministerial. Por ejemplo, la formación teológica en los seminarios y facultades de teología enfatiza la adquisición de conocimiento teológica y habilidades pastorales. No existe la posibilidad intrínseca a la formación teológica de desarrollar labores manuales o físicos.

La relación de profesor-alumno en muchos casos también se limita a los requisitos académicos exigidos a ambos. Esto a pesar de que la importancia de la influencia de los maestros en las vidas de los estudiantes ha sido reconocida como vital para el proceso educativo (Balmer, 2000). Varios factores, entre ellos la falta de tiempo, la ratio entre profesores y alumnos, así como el modelo de enseñanza catedrática pueden generar una distancia entre maestros y alumnos. Esto aleja a la formación ministerial actual del modelo ideal visualizado por Ellen White en base a las escuelas de los profetas.

Finalmente, se puede mencionar también la falta de planificación estratégica a la hora de ubicar los colegios e instituciones educativas a cargo de la formación teológica. Tal vez la facilidad de comunicación y transporte que existe en la modernidad no haga necesario buscar localizaciones estratégicas para los centros educativos. Sin embargo, también es posible que se produzcan 
menores oportunidades misioneras o se pierda influencia social al no buscar ubicaciones con mayor potencial.

\section{Conclusión}

En este breve artículo se han mostrado las características más importantes de las escuelas de los profetas del Antiguo Israel. En los inicios del sistema pedagógico adventista Ellen White, en su rol de propulsora y promotora de la educación cristiana, consideró que los principios en los que se basaban estas escuelas debían ser imitados en los colegios adventistas. Principalmente recalcó la importancia de contar con docentes consagrados que tenga una relación cercana y personal con sus alumnos, que el foco de la educación esté en el conocimiento de Dios y el desarrollo del carácter, que se busque el crecimiento holístico y balanceado de las tres áreas del ser (intelectual, físico y espiritual), que se den impartan habilidades manuales y oficios, y que la educación promueva la espiritualidad, la practicidad y la excelencia.

La sistematización académica de la filosofía adventista de la educación ha sido grandemente influida por los escritos de Ellen White quien, a su vez, formuló muchos de los postulados pedagógicos a partir de las escuelas de los profetas. Mediante este medio, estas escuelas han dejado su marca en la educación adventista y en la formación ministerial.

Algunas características del sistema pedagógico adventista que, directa o indirectamente, han sido inspiradas en las escuelas de los profetas son: el concepto holístico del ser humano y el desarrollo equilibrado del cuerpo, mente y espíritu; la búsqueda de la excelencia en los estudiantes; la utilización de profesores consagrados; y la aplicación de una enseñanza ministerial basada en la Escritura. Sin embargo, existen algunas características de las antiguas escuelas hebreas que aun no han podido aplicarse completamente en las instituciones educativas adventistas. La necesidad de un ambiente escolar cuasi familiar, con relaciones estrechas entre profesores y alumnos, la formación en oficios prácticos y la búsqueda de ubicaciones estratégicas para los centros educativos son puntos que aún quedan por ser alcanzados plenamente. Por lo tanto, las escuelas de los profetas aún tienen que ofrecer al sistema pedagógico adventista actual.

\section{Referencias}

Appiah, J., Danquah, E. \& Margo, E. K. (2017). Adventist Education in Ghana: Benefits, Challenges and Way Forward. Journal of AIIAS African Theological Association, 6, 11-27. 
https://www.academia.edu/33906979/ADVENTIST_EDUCATION_IN_GHANA_BENE FITS_CHALLENGES_AND_WAY_FORWARD

Balmer, B. (2000). The Teacher's Personal Touch: The Social Dimension in Adventist Education. Christ in the Classroom, 27, 1-18.

Becerra, E. (2008). Seventh-day adventist ministerial training: toward an integrated whole. Christ in the Classroom, $\quad 10, \quad 75-88$. http://circle.adventist.org/files/CD2008/CD1/ict/vol_10/10cc_075-088.pdf

Berman, J. A. (2017). Inconsistency in the Torah: ancient literary convention and the limits of source criticism. New York: Oxford University Press. https://doi.org/10.1163/15685330$\underline{12341367-01}$

Brown, W. J. (1972). Chronology of Seventh-day Adventist Education: Century of Adventist Education 1872-1972. Washington D.C.: Departamento de Educación de la Asociación General. $\quad$ https://education.adventist.org/wp-content/uploads/2017/10/Chronology-ofSeventh-day-Adventist-Education-1872-1972-Brown.pdf

Davidson, R. M. (2015). Schools of the prophet's paradigm for pastoral education. Current: Faith Meets Life and Culture, 19-22. https://www.academia.edu/19990976/Schools_of_the_Prophets_Paradigm_For_Pastoral_ Education

Dyrness, W. A. (1977). Themes in Old Testament Theology. Downers Grove, IL: InterVarsity Press.

Estep, J. R. (2003). Education among the Hebrews (2300-586 B.C.). En C.E.: The Heritage of Christian Education. Joplin, MO: College Press

Greenleaf, F. (2005). Timeline for seventh-day adventist education. Journal of Adventist Education, 67 (5), 10-15. http://circle.adventist.org/files/jae/en/jae200567051005.pdf

Hong, Y. P. (2000). The Schools of the Prophets: A Model for Alternative Adventist Education in $\begin{array}{lll}\text { Korea. Christ in } \quad \text { the } & \text { 99-112. }\end{array}$ https://christintheclassroom.org/vol_27/27cc_099-112.pdf

International Board of Ministerial and Theological Education (2017). Handbook of Seventh-day Adventist Ministerial and Theological Education. Silver Springs, MD: General Conference of Seventh-day Adventists.

Kaiser, W. C. (2001). The Old Testament Documents: Are they reliable and relevant?. Downer Grove, IL. InterVarsity Press.

Kido, E. (2010). For real education reform, take a cue from the Adventists. The Christian Science Monitor. $\quad$ https://www.csmonitor.com/Commentary/Opinion/2010/1115/For-realeducation-reform-take-a-cue-from-the-Adventists

Kitchen, K. A. (2003). On the Reliability of the Old Testament. Grand Rapids, MI: Eerdmans. 
Knight, G. R. (1980). Philosophy and Education: An Introduction in Christian Perspective. Berrien Springs, MI. Andrews University Press.

Knight, G. R. (1982). Issues \& Alternatives in Educational Philosophy. Berrien Springs, MI. Andrews University Press.

Knight, G. R. (1985). Myths in Adventism: An Interpretive Study of Ellen White, Education, and Related Issues. Hagerstown, MD: Review and Herald Publishing Association.

Knight, G. R. (2012). La filosofía de la educación: Segunda parte. Revista de Educación Adventista, 33, 24-41. https://education.adventist.org/wp-content/uploads/2017/10/JAE-Philosophyof-Adventist-Education-JAE-33-Spanish.pdf

Knight, G. R. (2015). The aims of adventist education: a historical perspective. Journal of Adventist Education, 77(4), 4-7. http://circle.adventist.org/download/AimsPerspective.pdf

Lemaire, A. (1981) Les écoles et la formation de la Bible dans l'ancien Israël. Göttingen: Vandenhoeck and Ruprecht. https://www.zora.uzh.ch/id/eprint/142958/1/Lemaire_1981_Ecoles_et_formation_de_la Bible.pdf

Matthews, J. V. G. (2014). A chesshire cat in the world of christian education. En Adventist Maverick: A Celebration of George R. Knight's Contribution to Adventist Thought. Nampa, ID: Pacific Press.

Millanao-Orrego, J. (1992). An Evaluation of the Concept of Seminary in Mission with Reference to the Latin American Adventist Theological Seminary (Tesis doctoral, Andrews University). https://digitalcommons.andrews.edu/dmin/200/

Peck, R. G. (1992). Theological Education in Context: A Pilot Extension Program for the Seventhday Adventist Church in Rwanda (Tesis doctoral, Andrews University). https://digitalcommons.andrews.edu/dmin/643/

Plaatjes, P. P. (2014). The nature, aims, and values of seventh-day adventist christian education. En: International Handbook of Learning, Teaching and Leading in Faith-Based Schools. Dordrecht: Springer. https://doi.org/10.1007/978-94-017-8972-1_22.

Provan, I., Long, V. P. \& Longman III, T. (2015). A Biblical History of Israel, 2da ed. Louisville, KY: Westminster John Knox Press.

Quinteros del Águila, F. (2013). Las escuelas de los profetas. Apuntes Universitarios, 3 (1), 105115. https://doi.org/10.17162/au.v0i1.295

Orwenyo, S. (2014). The Role of Theological Training in Pastoral Ministry within the Seventh-day Adventist Church: A Case Study of the Theological Seminary at the Adventist University or Africa. (Tesis de maestría, University of Nairobi). http://erepository.uonbi.ac.ke/handle/11295/95502 
Sicker, M. (2011). The First Book of Samuel: A Study in Prophetic History. Bloomington, IN: iUniverse.

Standish, C. y Standish, R. (1996). Education for Excellence. Rapidan, VA: Hartland Publications.

Taylor V, J. W. (2017). Schools of the Bible: contours of the divine plan for education. The Journal of Adventist Education, 79 (1), 13-26. https://jae.adventist.org/2017.1.3

Tayo, S., Dickson, T. U. y Josiah, U. G. (2019). Synergizing education and spirituality in faithbased tertiary institution. Journal of Education and Human Development, 8 (3), 64-75. https://doi.org/10.15640/jehd.v8n3a9

White, E. (1882). The schools of the prophets. Signs of the Times, 8 (24), 1-2 https://m.egwwritings.org/es/book/820.4498

White, E. (1885). The schools of the ancient hebrews. Signs of the Times, 11 (31), 1-2. https://m.egwwritings.org/es/book/32.397

White, E. (1890). Patriarchs and Prophets. Washington, D.C.: Review and Herald Publishing Association. https://m.egwwritings.org/en/book/84/toc

White, E. (1894). Christian Education. Battle Creek, MI: International Tract Society https://m.egwwritings.org/en/book/10.0

White, E. (1897). Special Testimonies on Education. https://m.egwwritings.org/en/book/103.0

White, E. (1901). Testimonios for the Church, vol. 6. Mountain View, CA: Pacific Press Publishing Association. https://m.egwwritings.org/en/book/118.0

White, E. (1903). Education. Mountain View, CA: Pacific Press Publishing Association. https://m.egwwritings.org/en/book/29.0

White, E. (1913). Counsel to Parents, Teachers, and Students. Mountain View, CA: Pacific Press Publishing Association. https://m.egwwritings.org/en/book/23.0

White, E. (1915). Gospel Workers. Washington, D.C.: Review and Herald Publishing Association. https://m.egwwritings.org/en/book/35.0

White, E. (1985). Spalding and Magan Collection. Payson, AZ: Leaves of Autumn Books. https://m.egwwritings.org/es/book/516.2

Williams, J. G. (1966). The prophetic "Father": A brief explanation of the term "sons of the Prophets". Journal of Biblical Literature, 85 (3), 344-348. https://doi.org/10.2307/3264248

Wood, L. J. (1990) Los profetas de Israel. Grand Rapids, MI: Portavoz. 